\title{
Interdisciplinary Project-based Learning in Ergonomics for Software Engineers: A Case Study
}

\author{
A. Branzan Albu K. Malakuti \\ University of Victoria \\ Dept. of Electrical and Computer Engineering \\ 3800 Finnerty Rd, Victoria (BC) Canada \\ aalbu@ece.uvic.ca
}

\begin{abstract}
This paper discusses an interdisciplinary educational initiative led by an instructional team with backgrounds in engineering and psychology in the context of an ergonomics course for software engineers. Our case study evaluates the educational outcomes of a course project that dealt with the error analysis and prototype-level redesign of a software tool for elderly users. The paper presents the rationale for the choice of this project, the project organization, and the evaluation of project-related outcomes with respect to the course learning objectives.
\end{abstract}

\section{Introduction}

There is an increasing need for interdisciplinary skills in engineers [6]. Such skills are in high demand in emerging career profiles, which integrate technological with nontechnological skills such as business knowledge, project management, and most importantly inter-personal communication. The academia's role is to prepare practitioners for such new societal demands. However, teaching interdisciplinary skills in undergraduate software engineering programs poses several challenges. Ertas et al [4] point out that 'disciplines inevitably develop into selfcontained hard shells' which encompass discipline-specific tools, methodologies, and jargon. With respect to the abovementioned communication skills, the highly-specialized intra-disciplinary jargon limits most the efficiency of interdisciplinary approaches to problem solving. Among all engineering specializations, software engineering ranks high in terms of interdisciplinary content. Knowledge from social sciences, psychology, and business is necessary as personal computing technologies and a large variety of software applications become ubiquitous. However, interdisciplinary skills are rarely taught in an explicit manner in

\author{
H. Tuokko W. Lindstrom-Forneri K. Kowalski \\ University of Victoria \\ Center on Aging \\ 3800 Finnerty Rd, Victoria (BC) Canada \\ htuokko@uvic.ca
}

software engineering courses. One possible reason for this is mentioned by Jaccheri and Sindre [5], who argue that adding interdisciplinary challenges to course projects may result in coursework overload and thus in reduced student performance. Our belief is that interdisciplinary course projects in software engineering need to be carefully formulated and planned in order to support and complement the theoretical content of the course. This close link between theory and practice should allow for maintaining a reasonable workload while maximizing the learning outcomes of the course. This paper presents an example of such an interdisciplinary course project for Ergonomics, which is a senior-year course in the Software Engineering program at the University of Victoria, Canada. Our objective is to investigate whether this interdisciplinary course project can help achieve the learning objectives of the course. The remainder of the paper is structured as follows. Section 2 outlines the methodology used in the construction and implementation of this interdisciplinary initiative. Section 3 provides an evaluation of the project-related outcomes with respect to the course learning objectives. Section 4 draws conclusions and describes future work.

\section{Methodology}

This section describes the proposed project in the context of the Ergonomics course and discusses the relevance of the project for the learning outcomes of the course. Contextual information about the Ergonomics course content is presented first. Next, we present the description and in-class implementation of the project.

\subsection{Ergonomics for Software Engineers: Course Overview}

The Ergonomics course at the Faculty of Engineering (UVic) addresses the need for software engineers to inte- 
grate knowledge of human strengths and limitations in system design and analysis. As a result of taking this course, students should be able to:

- C.O.1 Understand and know how to apply the usercentered design paradigm

- C.O.2 Know how to perform a user and task analysis prior to product design/redesign

- C.O.3 Design displays and controls which are compatible to human perception and attention mechanisms

The course objectives (C.O.) listed above show the interdisciplinary nature of the Ergonomics course content. For instance, focus on the user integrates knowledge from psychology (perception mechanisms, cognitive modeling), social sciences (designing user interviews and questionnaires), and system design (iterative prototyping). Our belief is that such interdisciplinary concepts are best learned via an experiential approach, or learning-by-doing. This approach was implemented via a new interdisciplinary project which is described below.

\subsection{Project overview}

Project-based learning is a popular education method in engineering curricula, as shown in [8], [3], [7]. Projects represent the hands-on component of engineering courses, where students apply theoretical knowledge in the context of a specific application. However, such projects rarely exhibit interdisciplinary content [5], even when such content would support best the theoretical component of the course. The proposed project addresses this problem via a collaborative teaching scheme for interdisciplinary projects.

\subsubsection{Project theme selection}

The project consisted in the prototype-level redesign of a software tool for older users. This software, called AAA Roadwise Review [9], was designed for the home-based self-assessment of driving abilities in older drivers. It contains a battery of computer-based tests for the assessment of physical and cognitive driving-related skills. The tests composing Roadwise Review can be seen in Figure 1. While of most of these tests are computer-based, the Leg Strength and General Mobility test, as well as the Hand-Neck Flexibility use the computer only for test-related instructions, timing, and data storage, and require a partner for test completion.

User testing sessions conducted at the Center on Aging (UVic) prior to this project revealed several usability flaws in Roadwise Review. We considered the redesign of Roadwise Review as an ideal Ergonomics project theme for the following three reasons. First, software (re)design for older

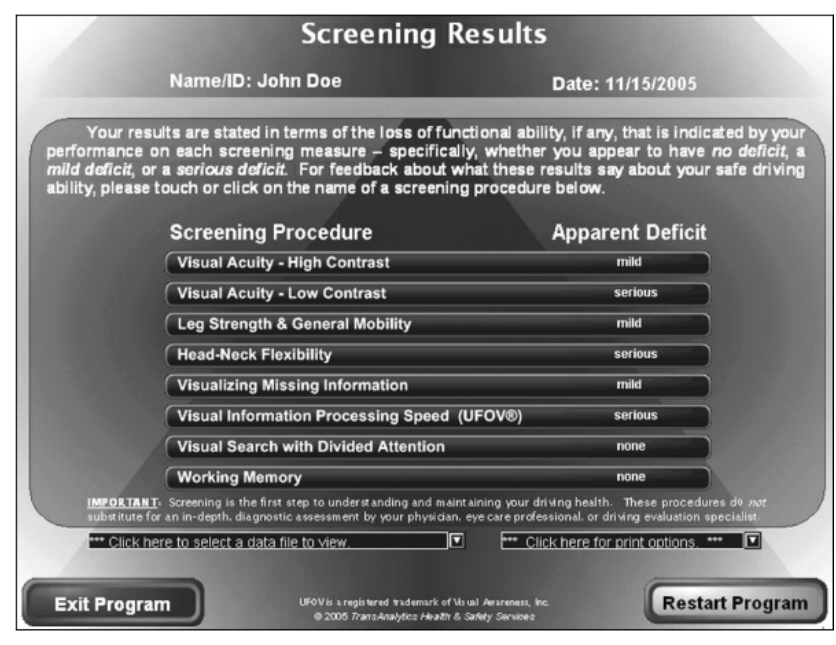

\section{Figure 1. Results page showing the battery of tests [9]}

users offers a good example for applying a fundamental law in human factors of interactive software: "the user is not you!" . In order to provide an efficient redesign, students needed to perform a thorough user analysis. Second, flaws identified in user testing sessions went beyond user interface issues, thus challenging students to address a variety of ergonomic problems including interface (re)design, but also software delivery methods and information management. Third, although the organization of testing sessions with elderly users for the redesigned prototypes was not possible due to the rigid timeframe of a course project, user focus was significantly facilitated by the expertise in aging existent in our instruction team.

\subsubsection{Project requirements}

The project deliverables consisted in a prototype version of the redesigned software to be demonstrated in class, and in a technical report. The successful completion of the project involved the fulfillment of the following requirements:

- Identification and discussion of ergonomic design flaws in the Roadwise Review software. The identification process had to be based on a heuristic evaluation of the software, as well as on quantitative and qualitative results that were previously obtained from user testing sessions. The discussion had to explain each identified flaw via ergonomic concepts and interface design guidelines.

- Generation of prototype-level redesign solutions for the above-identified flaws. In order to insure a reasonable project-related workload, and to direct the students' attention to conceptual redesign, horizontal pro- 
totypes were requested (i.e. with high fidelity in look and feel, and low-fidelity in depth, or functionality).

- Justification of the implemented redesign solutions using ergonomic concepts. This discussion had to mirror the discussion of identified flaws and to be structured along the user-centric design paradigm.

The above requirements show that this project places equal emphasis on design as well as on the development of analytical and reflective skills. While design has been already identified as a central component in interdisciplinary learning [4], the development of analytical and reflective practice skills is not discussed in related work. We consider that the development of such skills is greatly facilitates integration of theoretical and applied (project-based) knowledge taught in engineering courses.

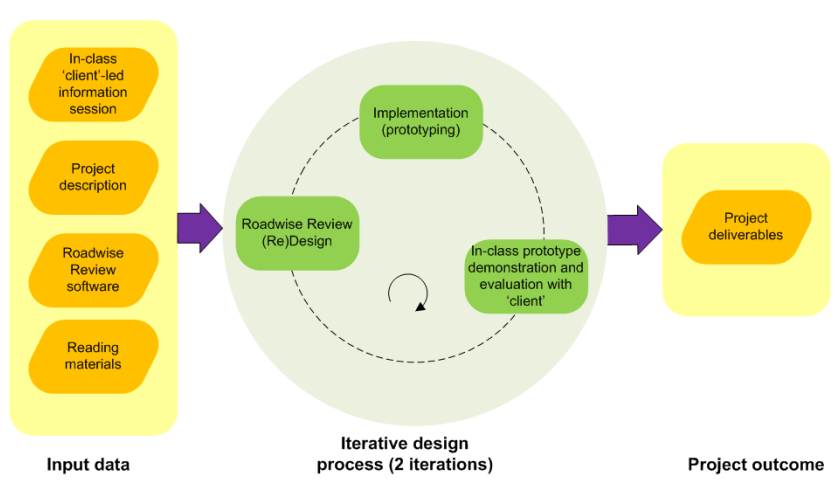

Figure 2. The process of project development

\subsubsection{In-class project development}

The in-class project development is modeled as an iterative process, as shown in Figure 2. All modules in this figure are described below, with the exception of the project deliverables which were discussed in 2.2.2. The input data consists in project-related information that was made available to students at the beginning of the project. Students attended first a 90 minutes in-class information session on aging-related driving issues. This presentation was conducted by the members of our team with expertise in aging, who acted as 'clients' throughout the redesign project. Contextual information about older adults and driving, and about the safety of elderly drivers was presented first, in order to convey the rationale of creating software tools for the self-assessment of driving abilities. Next, the Roadwise Review software was briefly overviewed with recommendations for further self-study of reading materials and of the software itself. The session ended with some examples of usability issues discovered during the user testing sessions, while mentioning that students were expected to identify and correct as many usability issues as possible in their project work. The take-home message of this information session was that usability issues have a significant impact on the efficiency of the self-assessment tool, and that the redesign of Roadwise Review had to address these issues by adopting a user-centric design approach. Project-related reading materials included [9], which presents the content and initial design of Roadwise Review, and [2] which offers insight into ageism and technology issues. A summary of qualitative data gathered during previous user testing sessions of Roadwise Review was also made available. These readings were selected as a logical follow-up of the in-class information session, as they provide a solid background for the start of the redesign process [9] and for the user profiling ([2] and qualitative user testing data). The Roadwise Review software was provided to each project team in CDROM format. All teams received the same project description, and inter-team collaboration was discouraged by organizing the final prototype demonstrations as a marked competition. The redesign process had to follow an iterative scheme which involved client feedback at each iteration. The iterative design is a specialization of a spiral model described in a seminal article by [1]. We selected this iterative design approach since we found it suitable for encouraging interdisciplinary developer-'client' communication.

\section{Discussion of project-related outcomes}

This section investigates whether the project outcomes support the achievement of the course learning objectives outlined in 2.2.2. Our study is performed upon the projects of student teams who have all agreed to have their redesign ideas shared with experts in aging and in software design. The students' perspective on the usefulness of this interdisciplinary learning experience was captured via anonymous post-project questionnaires. Table 1 shows the percentages of respondents that found the project useful for the achievement of each course objective specified in Section 2.2.

\begin{tabular}{|l|l|l|l|}
\hline Course objectives & C.O. 1 & C.O. 2 & C.O. 3 \\
\hline Percentage of respondents & $60 \%$ & $80 \%$ & $75 \%$ \\
\hline
\end{tabular}

\section{Table 1. Percentage of respondents that found the Roadwise Redesign project useful for achieving the course objectives}

The remainder of this section discusses the educational value of the Roadwise Review redesign project from the perspective of the instructional team who authors this paper. We consider that this interdisciplinary project has fulfilled its purpose, which is supporting the achievement of course 
objectives via an experiential learning approach. Our statement is justified in the following subsections by a detailed analysis of the outcomes of user and task analysis steps, as well as of the main (re)design ideas in relationship to course objectives. Examples of team-specific results are also provided.

\subsection{User-centric design}

The first course objective outlined in 2.1 concerns the understanding and application of the user-centric design paradigm. We found that this paradigm was instantiated in all projects in various ways. Across all projects we have identified one major instance, namely the creation of personas and scenarios from user testing data and literature on aging. An example of persona and its associated scenario is given in the project excerpt below.

"[Persona] Name: Edna; Age: 68; Ethnicity: Caucasian; Marital status: Widow; Profession: Retired/Hobby Farm; Education: High school;

After working for many years as a bank teller, Edna is now retired. She spends her days raising goats and chickens on her hobby farm located in the Lower Mainland. Edna's husband, Wallace, drove Edna and himself into town regularly for food, farm supplies, and to visit and care for Edna's elderly mother, Rita, who still lives at home on her own, even at the ripe old age of 89. Recently, however, Wallace passed away due to sudden heart failure. Because she has driven so little over the past several years, Edna is unsure of her ability to drive into town on a regular basis. She has also developed severe arthritis since she last drove regularly. Edna is now living alone on the hobby farm that she and Wallace maintained. She wishes to continue running the farm. In order to do so, regular trips into town will be needed to pick up supplies for herself and for the animals. Edna also needs to visit her elderly mother, who lives alone and doesn't go out by herself anymore. Edna will need to drive her mother around for groceries, doctor appointments, and the like. This has caused her to feel especially concerned about her driving skills, as she does not want to be involved in an accident while her mother is a passenger.

[Scenario] Due to her lack of recent driving experience, and her advanced arthritis, Edna decided to purchase the Roadwise Review in order to test her abilities. Edna is not very knowledgeable with computers and so she asked her son, Charles, to aid her with using the software. Despite her arthritis, Edna was able to progress through the various tests, although somewhat slowly. However, when starting one of the later tests, the program suddenly froze. Unable to get the program to respond, she had to press the reset button on her computer, and in so doing lost the results of all the tests she had completed thus far. Frustrated that she was not able to complete all tests, and angry at her computer -which she never really liked using to begin with-Edna refused to sit through the entire test process again, and never finished the Roadwise Review." The excerpt above is an example of creating a persona by aggregating interdisciplinary information (social aspects of aging, decline of driving abilities in older drivers, attitudes related to driving and computer use). Other personas embodied user characteristics that were relevant for the interaction with Roadwise Review such as:

- variable degree of computer experience, ranging from none to advanced. This feature is relevant for the redesign process, since users who are familiar with computers would not need the mandatory mouse practice included in Roadwise Review.

- physical characteristics: some personas exhibited a limited set of health problems, for instance only vision-related. For such users, the rigid sequencing of tests in Roadwise Review is not efficient.

The scenario in the excerpt above was inspired from a critical incident (loss of test results due to software crash and to monolithic structure of software) that was experienced by most of the teams during software walkthroughs. Other scenarios included critical incidents that were inspired from qualitative data gathered during user testing sessions such as:

- difficulties encountered during the use of the help module, which explains only the rationale of each test, without providing any instructions on how to perform the test;

- frustrating experiences related to having to watch the entire video in order to get specific information about how to perform the test etc.;

We found that the creation of personas and scenarios was a useful exercise in user analysis, as it involved aggregating interdisciplinary information from multiple sources. Moreover, scenarios provide a starting point for the task analysis process, which is detailed below.

\subsection{Task analysis and display/control re- design}

Knowing how to perform a task analysis is part of the second course objective outlined in 2.1, while display/control redesign is addressed by the third course objective. Scenarios can be considered anchor points of task analysis, since they are narrative constructs that enliven results from the qualitative analysis of the user testing sessions. Each scenario offers a realistic glimpse of a critical incident likely to occur during the use of Roadwise Review. 
However, a set of scenarios fails to draw a comprehensive portrait of the software flaws due to its unordered structure. This is why the project required performing task analysis via cognitive walkthroughs and heuristic evaluation protocols. While task analysis follows strict procedures in software engineering, it also integrates user domain knowledge in order to correctly model user actions. This interdisciplinary dimension of task analysis is particularly important for the successful redesign of Roadwise Review. Major design errors that were identified in all projects via task analysis include the navigational model and the user support (help). Details about these two issues follow below. The navigational model in Roadwise Review is linear and does not allow users a random access to tests. The software lacks elementary navigational controls such as 'back' and 'forward' buttons. These limits are addressed in redesigned navigation models such as the one shown in Figure 3. This specific solution is based on an internet-based implementation of the software which would eliminate the task of installing the software prior to its use. Regarding the help module, cognitive task analysis revealed the lack of controls for the rate and amount of information presented in tutorial videos/textual help. Moreover, users are not able to go back to instructions once having entered the test. The redesign solutions emphasize that the help section should provide users with answers to any questions that they may have during the use of the software. This finding is consistent with the necessity of providing continuous memory support for elderly users. One example of redesign divides the display between test zone and instructions zone, as shown in Figure 3.

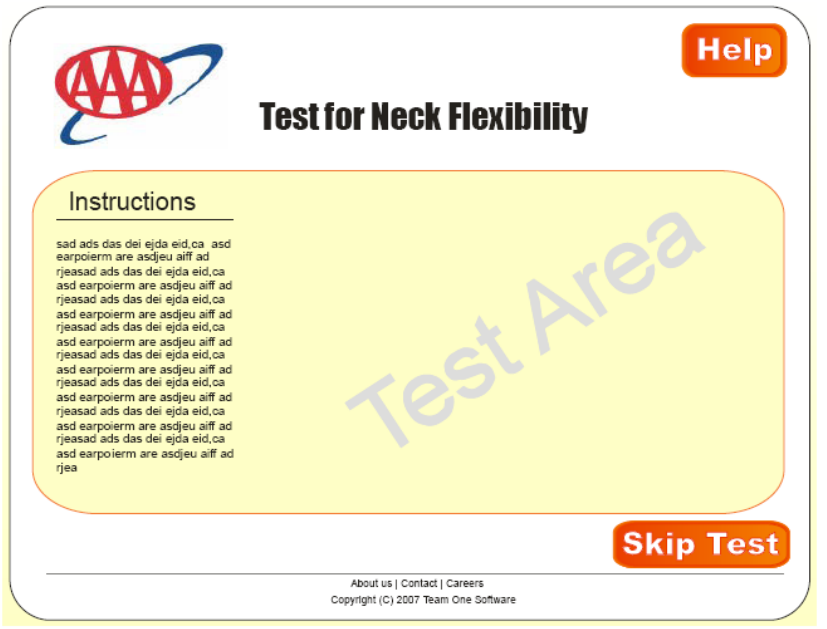

Figure 3. Redesigned display that supports user memory by maintaining instructions on screen

\section{Conclusions}

The presented case study is an example of a successful collaboration between Engineering and Psychology disciplines in the area of teaching Ergonomics for Software Engineers. The collaboration was considered successful since the project outcomes fully supported the achievement of the course learning objectives. The proposed course project had elements that 'forced' students in software engineering to focus on interdisciplinary areas related to ergonomic software design in the context of a very specific application. Such areas include aging, human perception mechanisms and human information processing in older adults, and software design for computerized psychological tests. This pilot study has demonstrated the educational value of interdisciplinary projects for Ergonomics. Future course projects will be designed in a manner that will increase students' exposure to user domain knowledge by ethnographic studies for user and task analysis. Specifically, we intend to replace the 'shortcut' that provided summarized results from pre-project user testing sessions with observational studies to be performed by students on real users during software use. Moreover, although the proposal of one single theme has facilitated the inter-team comparison of project results, we aim at providing students with choices between various project themes. This choice will allow for using personal interests as an intrinsic motivational factor in increasing students' performance on projects.

\section{Acknowledgments}

We acknowledge the contributions to redesign aspects of Roadwise Review of the class of SENG 4122007 at the University of Victoria, and in particular of Trevor Beugeling (who wrote the persona and scenario shown in this paper) and of the team composed of Tyrone Austen, Andre Campos, Royer Henaine, Mani Mobini, Ali Yousefi whose work was showcased in Figures 3, 4.

\section{References}

[1] B. Boehm. A spiral model of software development and enhancement. IEEE Computer, 21(5):61-72, 1988.

[2] S. Cutler. Ageism and technology. Generations, American Society on Aging, 29(3):67-72, 2005.

[3] D. Davenport. Experience using a project-based approach in an introductory programming course. IEEE Transactions on Education, 43(4):443-448, 2000.

[4] A. Ertas, T. Maxwell, V. Rainey, and M. Tanik. Transformation of higher education: The transdisciplinary approach in engineering. IEEE Transactions on Education, 46(2):289295, 2003.

[5] L. Jaccheri and G. Sindre. Software engineering students meet interdisciplinary project work and art. In Proc. of 11th 


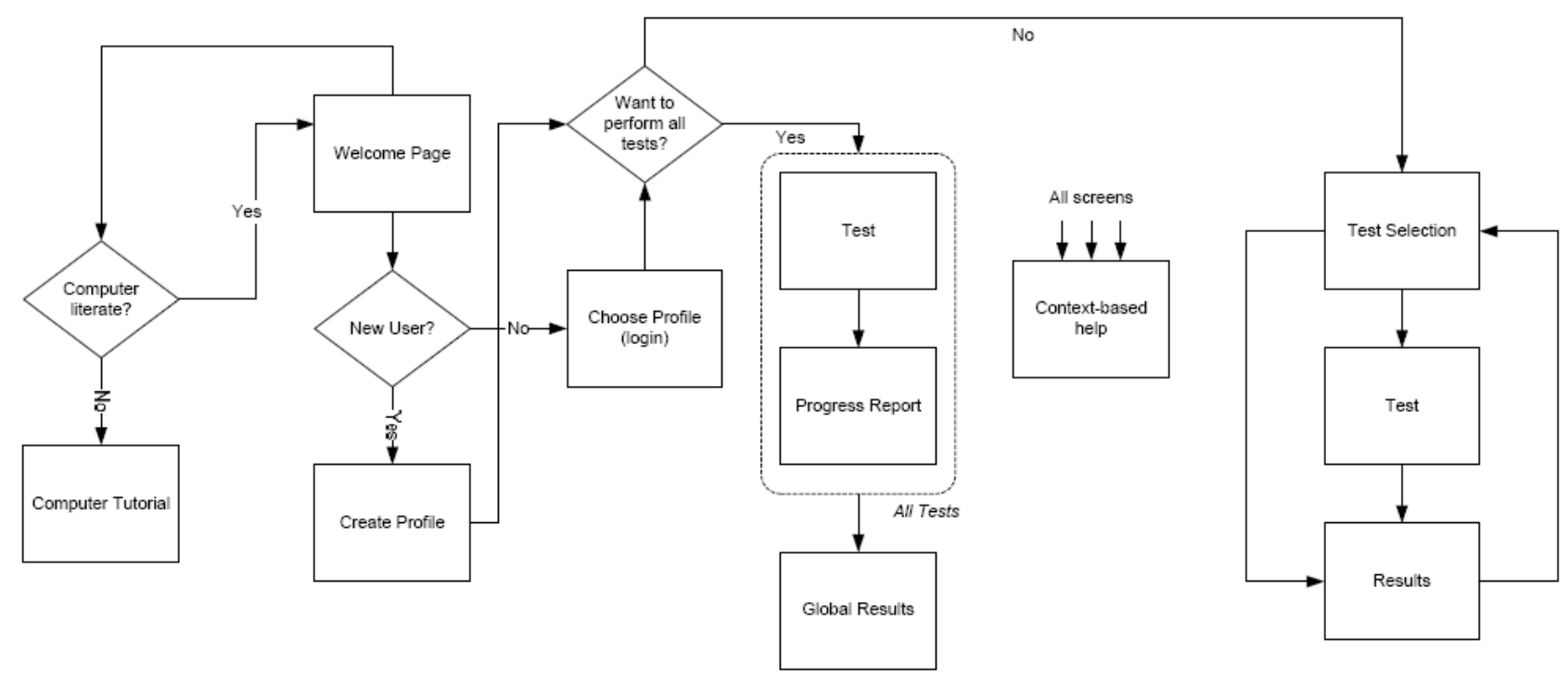

Figure 4. Redesigned navigational model for Roadwise Review

IEEE International Conference on Information Visualization (IV'07), 2007.

[6] Y. Julliard and A. Schwab. The role of discourses in multidisciplinarity. In Proc. IEEE International Symposium on Technology and Society (ISTAS'01), Stanford, US, 2001.

[7] J. Macias-Guarasa, J. Montero, R. San-Segundo, A. Araujo, and O. Nieto-Taladriz. A project-based learning approach to design electronic systems curricula. IEEE Transactions on Education, 49(3):389-397, 2006.

[8] A. R. M.L. Ho and P. Chan. Project-based learning. IEEE Control Systems Magazine, pages 88-91, 2004.

[9] L. Staplin and T. B. Dinh-Zarr. Promoting rehabilitation of safe driving abilities through computer-based clinical and personal screening techniques. Topics in geriatric rehabilitation, 22(2):129-138, 2006 